\title{
网格模型上基于线性插值的自适应保特征去噪算法
}

\author{
岳少阳 ${ }^{1)}$, 李楠楠 ${ }^{1)}$, 王为莹 ${ }^{1)}$, 王辉 ${ }^{2)}$, 包敏泽 ${ }^{1)}$, 蒋波 ${ }^{1 *}$ \\ ${ }^{1)}$ (大连海事大学信息科学技术学院 大连 116000) \\ ${ }^{2)}$ (石家庄铁道大学信息科学技术学院 石家庄 050000) \\ (nannanli@dlmu.edu.cn)
}

\begin{abstract}
摘 要: 网格去噪是改善扫描设备所得数据并为后续数字几何处理提供理想模型的重要步骤. 针对近年来涌现出的 各种网格去噪算法难以平衡好时间消耗和去噪结果之间的关系, 提出一种高效的基于线性插值的框架性保特征去噪 算法, 将体现结构特征的充分磨光模型与包含综合信息的噪声模型进行联合分析，通过自适应的线性插值算法逐步 从噪声模型中提取出特征信息加到光滑模型中，从而实现保持不同程度特征(从显著特征到微弱特征)的去噪结果. 同时提出迭代去噪算法的迭代终止判别条件，可为不同迭代去噪算法提供自动且可靠的终止条件. 大量在不同复杂 度和不同噪声程度的模型上的实验结果表明, 与现有的局部迭代算法相比, 该算法取得更好的视觉效果和较低的均 方角度误差值，能够保持不同程度的特征且具有较好的时间性能.
\end{abstract}

关键词：网格去噪；线性插值；保特征；不同程度的特征；自适应设置

中图法分类号: TP391.41 DOI: 10.3724/SP.J.1089.2020.18104

\section{Linear Interpolation Based Adaptive Feature-Preserving Filtering Method on Mesh Models}

\author{
Yue Shaoyang ${ }^{1)}$, Li Nannan ${ }^{1)}$, Wang Weiying ${ }^{1)}$, Wang Hui ${ }^{2)}$, Bao Minze ${ }^{1)}$, and Jiang Bo ${ }^{1{ }^{*}}$ \\ 1) (School of Information Science and Technology, Dalian Maritime University, Dalian 116000) \\ ${ }^{2)}$ (School of Information Science and Technology, Shijiazhuang Tiedao University, Shijiazhuang 050000)
}

\begin{abstract}
Mesh denoising is an important step to improve the data obtained by scanning devices and it provides an ideal model for subsequent digital geometry processing. The main challenge is to remove the noises while maintaining its geometric features, especially weak features. The denoising methods occurred in recent years oftentimes lack the capability to balance between time consumption and denoising results. Therefore, this paper proposes an efficient feature preserving denoising framework based on linear interpolation. In order to realize feature-preserving (for features of different levels, from salient features to weak features) denoising, this paper uses the interpolation method to jointly analyze the fully polished model that reflects the structural features and the noisy model that contains the comprehensive information. By designing an adaptive interpolation method, the features of different levels are extracted from the noisy model and added to the smooth model. At the same time, an automatic termination judgment for the iterative denoising algorithm is proposed, which provides automatic and reliable termination conditions for different iterative denoising al-
\end{abstract}

收稿日期: 2019-09-30; 修回日期: 2020-02-04. 基金项目: 国家自然科学基金(61802045, 61972267); 虚拟现实技术与系统国家重点 实验室(北京航空航天大学)开放课题基金(VRLAB2020A04); 大连市科技创新基金(应用基础研究)(2018J12GX042); 河北省自然科学基 金(F2018210100); 河北省高等学校青年拔尖人才项目(BJ2018003). 岳少阳(1993-), 男, 硕士研究生, 主要研究方向为计算机图形学; 李楠楠(1987一), 女, 博士, 讲师, 硕士生导师, CCF 会员, 主要研究方向为计算机图形学、深度学习、计算机视觉; 王为荣(1995一), 女, 硕士研究生, 主要研究方向为计算机图形学; 王辉 (1983-), 男, 博士, 副教授, 硕士生导师, CCF 会员, 主要研究方向为计算机图形学; 包敏泽(1994一), 男, 硕士研究生, 主要研究方向为计算几何; 蒋波(1960一), 男, 硕士, 硕士生导师, 论文通讯作者, 主要研究方向为软 件技术与算法、软件理论与软件工程、计算几何. 
gorithms. The quantitative and qualitative results on synthetic and real data with various levels of noises show that our method can achieve efficient and robust denoising results.

Key words: mesh denoising; linear interpolation; feature-preserving; different levels of features; adaptive settings

\section{1 介 绍}

随着数字几何应用的不断拓展，其对三维模 型的需求量与日俱增, 但是在使用三维扫描设备 (如三维摄像机和激光扫描仪等)获取三维模型的 过程中，模型不可避免地会被引人不同复杂程度 的噪声. 因此, 网格模型去噪是数字几何处理的重 要步骤. 网格去噪算法的主要目的是能够在去除 模型上噪声的同时保留或者恢复模型自身的特征, 并且不引入额外的噪声. 网格去噪算法根据模型 点位置、点法向和面法向等几何信息对模型的特征 位置进行分析与识别. 由于模型的形态差异较大、 噪声程度难以判别, 现有算法往往采取多步骤或 者多次迭代的方式进行处理，使得算法的时间复 杂度较高, 并且需要大量地调参、终止迭代等手工 操作. 因此, 本文提出一套基于线性插值的框架性 去噪算法.

在三维去噪问题中, 最早提出的是局部迭代 算法 ${ }^{[1-12]}$, 其特点是能够较好地利用模型的邻域信 息来描述模型局部细节特征. 局部算法需要将卷 积操作扩散到全模型, 但是依赖于较多次数的迭 代. 当迭代次数过多时, 弱特征会被去除, 而当迭 代次数过少时, 则不能很好地去除噪声. 因此需要 人为参与卷积函数方差选取与迭代步骤设定. 相 比之下，全局算法 ${ }^{[3-18]}$ 不需要迭代，且由于其基于 模型的全局优化而能够保持模型的整体结构, 但 是此类算法中卷积方差往往是全局统一设定的, 导致细节特征不能被很好地保持. 为了提升局部 和全局算法的性能，一些工作 ${ }^{[19-21]}$ 倾向于在全局 算法或者局部算法的基础之上加人一步或者更多 的预处理或者后处理步骤. 例如，特征探测和子邻 域搜索等. 多步骤算法虽然能够通过特征探测识 别特征与噪声, 但是处理步骤过多, 需要调节的参 数过多, 算法的时间复杂度较高. 最新的网格去噪 工作主要借助于深度学习工具 ${ }^{[22-23]}$, 并取得显著 效果, 但是此类算法训练过程需要消耗大量的时 间, 而且去噪学习的泛化能力还比较差, 很难恢复 没有被包含在训练集中的特征. 综上所述，如何简 化三维模型去噪过程、提出一种鲁棒高效的算法是
一个亟待解决的问题.

由于局部去噪算法能够较好地保持模型的局 部特征, 但随着迭代进行一些微弱特征被逐步去 除, 较多研究工作尝试在迭代的过程中逐渐地加 人特征来改进其性能. Wang 等 ${ }^{[5]}$ 在双边滤波 ${ }^{[3]}$ 的基 础上, 应用滚动指导滤波来进行改进, 为了达到挖 掘噪声中隐藏的特征信息, 在每一次迭代的过程 中将原始法向作为卷积对象, 而不是反复对上一 步所得法向进行卷积. 但是该算法不是自适应的, 不能根据每个三角形面片的情况做差异化处理. 两步骤的算法 ${ }^{[15]}$ 先用全局的拉普拉斯规范得到基 底模型，再用压缩感知从基底模型和输人模型的 残差中恢复特征. 由此可见, 原始噪声网格中隐含 着模型的噪声信息; 因此, 本文希望用原始噪声模 型和充分磨光模型进行插值、逐渐提取噪声模型中 的特征加到光滑模型中.

综上，本文提出一种基于线性插值的、快速高 效的网格模型去噪框架性算法, 其总体来看是将 去噪模型看做噪声模型和充分磨光模型的中间插 值结果. 之所以说其为框架性算法, 是因为其中间 环节中的充分磨光模型获取算法和插值算法皆可 替换. 本文通过设计自适应的插值函数, 将噪声模 型上的特征逐步提取出来加到充分光滑模型上. 其中插值函数的设计主要基于如下观察: 在局部 迭代的过程中, 光滑区域的法向应趋于稳定不变, 特征区域的法向信息应逼近噪声模型中隐含的特 征信息. 进一步地, 而且通过设计迭代终止条件, 有效地避免现有迭代去噪算法人工控制迭代过程 的问题，在线性插值提升效率的基础上进一步简 化去噪步骤.

\section{2 相关工作}

\section{1 全局和局部算法}

局部算法是去噪中比较经典的算法, 鉴于面 法向比顶点位置能更好地表示局部几何信息, 通 常研究人员通过面法向进行滤波后的法向来更新 点的位置, 以得到去噪后的曲面. 局部算法对法向 的处理通常是使用卷积函数对三角形面片与邻域 
法向几何信息的差异进行加权取均值达到去噪的 目的. 研究人员考虑使用三角形面片不同的几何 信息 ${ }^{[1,3,10]}$ 或者改进卷积核函数 ${ }^{[11-12]}$ 来提升去噪性 能. Fleishman 等 ${ }^{[1]}$ 和 Zheng 等 ${ }^{[3]}$ 的算法都是对图像 双边滤波的延伸. Fleishman 等 ${ }^{[1]}$ 考虑顶点位置局 部差异和点法向差异来做高斯卷积, Zheng 等 ${ }^{[3]}$ 考 虑的是质心距离和法向差异来做高斯卷积，与文 献[1]相比它们对局部细节特征处理得更加细致. 与之前常规滤波算法考虑模型法向信息和顶点信 息不同, Centin 等 ${ }^{[10]}$ 使用模型曲率信息，通过局部 曲率变化和总体曲率变化更新法向, 以保持和增 强模型的特征. 通过改变卷积核函数来改变法向 权重也是改进方式之一，Liu 等 ${ }^{[11]}$ 提出在测地距上 计算法向差异, Yadav 等 ${ }^{[12]}$ 通过改进的高斯卷积核 函数来阻止尖锐边区域的扩散. Zhang 等 ${ }^{[4]}$ 首先在 原始模型上提取出指导信号，然后利用指导信号 来改进双边权重的设计. Wang 等 ${ }^{[5]}$ 在 Zheng 等 ${ }^{[3]}$ 基 础之上提出滚动指导滤波 ${ }^{[6]}$, 迭代过程中每一步都 用原始的法向作为指导，更好地保持了原始模型 的特征. Liu 等 ${ }^{[7]}$ 在指导滤波 ${ }^{[4]}$ 的基础上，通过特征 探测识别特征面与非特征面, 并提出邻域选择的 度量来增强特征，取得比指导滤波更好的效果. 滤 波过程中对邻域面片进行选择 ${ }^{[2,8-9]}$ 的处理同样可以 提升效果. Sun 等 ${ }^{[2]}$ 根据三角形面片与邻域面片法向 角度选择邻域, $\mathrm{Li}$ 等 ${ }^{[8]}$ 在滤波过程中对尖锐特征使 用基于边的邻域处理, Hurtado 等 ${ }^{[9]}$ 选择邻域除了点 和法向信息外考虑梯度信息来处理邻域. 局部算法 能够通过迭代很好地逐渐恢复特征，但为了保持细 节特征，局部算法的迭代次数往往很难自动设定.

基于优化模型的全局去噪算法近些年也受到 广泛关注. Zheng 等 ${ }^{[3]}$ 提出基于双边权拉普拉斯的各 向同性去噪算法，但是拉普拉斯无法区分微弱特 征与噪声, 并且无法处理好大噪声模型. 基于边缘 算子的网格去噪算法 ${ }^{[13]}$ 和结合分段常量函数和总 体变化的变分网格去噪算法 [16]都可以解决文献[3] 中算法存在的问题，但是对于最后模型的光滑弧形 区域总是存在不理想梯状效果，特别是 $\mathrm{He}$ 等 $^{[13]}$ 的 算法. Zhao 等 ${ }^{[14]}$ 对非凸求解过程进行优化，提出来 一个改进的交替策略来求解最小问题. Wang 等 ${ }^{[15]}$ 从 用全局拉普拉斯规范得到的基底网格和原始网格的 残差中通过压缩感知恢复特征。二阶规范化 ${ }^{[17-18]}$ 在多种去噪算法得到应用, Liu 等 ${ }^{[17]}$ 的算法将二阶规 范化与法向保真项相结合; Zhong 等 ${ }^{[18]}$ 在文献[17] 的基础之上，考虑总体变分差异和法向的保真，更 好地保持了模型的细节. 全局算法能够较好地保
留整体结构, 但是并不能很好地保持局部细节。

\section{2 其他去噪算法}

为了提升局部和全局算法的性能,一系列混 合使用全局滤波、局部滤波、中值滤波、特征探测、 子邻域搜索和点的滤波等的多步骤算法 ${ }^{[19-21]}$ 被提 出. 因为多步骤类算法处理细致, 所以取得了较好 的效果. 但是它们均存在计算复杂度较大、参数过 多等问题.

深度学习的算法 ${ }^{[22-23]}$ 也被应用到去噪问题中. Wang 等 ${ }^{[22]}$ 提出通过滤波面法向描述子, 从噪声网 格面邻域到没有噪声网格面法向中的映射形成非 线程的回归函数, 使用回归函数来计算新的面法 向. Wang 等 ${ }^{[23]}$ 提出先从噪声模型和真实模型对中 学习, 使用噪声差异通过神经网络来移除噪声, 然 后从滤波模型和真实模型通过回归函数恢复特征. 深度学习的算法取得一定的效果, 但是训练过程 是耗时的, 并且训练结果受数据集的影响, 没有被 包含在训练数据集中的特征是不能恢复的.

\section{3 经典去噪算法与知识准备}

网格上的去噪算法由双边滤波算法开始并围 绕其展开, 所以本节首先介绍经典的双边滤波 ${ }^{[3]}$, 然后介绍其改进算法滚动指导滤波 ${ }^{[5]}$, 也以此展开 对相关知识的介绍, 为后续章节做准备.

给定一个三角网格模型, 记网格 $\boldsymbol{M}=(\boldsymbol{V}, \boldsymbol{E}, \boldsymbol{F})$, 其中, $\boldsymbol{V}, \boldsymbol{E}$ 和 $\boldsymbol{F}$ 分别表示网格的顶点、边和面的集 合. 以 $\boldsymbol{c}_{i}$ 表示三角面片的质心, $\boldsymbol{n}_{j}$ 表示三角形面片 的原始法向, $\boldsymbol{P}_{i}$ 表示三角形面片的邻域， $\varepsilon_{i j}$ 表示三 角形面片的采样率(一般取值三角形面片的面积), 采样率能够改进不规则的面采样模型的去噪效果.

双边滤波算法可以表示为

$$
\boldsymbol{n}_{i}^{t+1}=\frac{\sum_{j \in \boldsymbol{P}_{i}} \boldsymbol{W}_{\mathrm{c}}\left(\left\|\boldsymbol{c}_{i}-\boldsymbol{c}_{j}\right\|\right) \boldsymbol{W}_{\mathrm{s}}\left(\left\|\boldsymbol{n}_{i}^{t}-\boldsymbol{n}_{j}^{t}\right\|\right) \varepsilon_{i j} \boldsymbol{n}_{j}^{t}}{\sum_{j \in \boldsymbol{P}_{i}} \boldsymbol{W}_{\mathrm{c}}\left(\left\|\boldsymbol{c}_{i}-\boldsymbol{c}_{j}\right\|\right) \boldsymbol{W}_{\mathrm{s}}\left(\left\|\boldsymbol{n}_{i}^{t}-\boldsymbol{n}_{j}^{t}\right\|\right) \varepsilon_{i j}}
$$

其中, $\boldsymbol{W}_{\mathrm{s}}$ 和 $\boldsymbol{W}_{\mathrm{c}}$ 分别为面心(三角面片的质心)距离 和面法向差异的高斯卷积函数, 它们分别定义为

$$
\left\{\begin{array}{l}
\boldsymbol{W}_{\mathrm{c}}\left(\left\|\boldsymbol{c}_{i}-\boldsymbol{c}_{j}\right\|\right)=\exp \left(\frac{-\left\|\boldsymbol{c}_{i}-\boldsymbol{c}_{j}\right\|^{2}}{2 \sigma_{\mathrm{c}}^{2}}\right) \\
\boldsymbol{W}_{\mathrm{s}}\left(\left\|\boldsymbol{n}_{i}^{t}-\boldsymbol{n}_{j}^{t}\right\|\right)=\exp \left(\frac{-\left\|\boldsymbol{n}_{i}^{t}-\boldsymbol{n}_{j}^{t}\right\|^{2}}{2 \sigma_{\mathrm{s}}^{2}}\right)
\end{array}\right.
$$

其中, $\sigma_{\mathrm{s}}$ 和 $\sigma_{\mathrm{c}}$ 为高斯函数的方差参数, $\sigma_{\mathrm{c}}$ 通常被 设定为所有邻接三角面片面心的平均距离; $\boldsymbol{n}_{j}^{t}$ 表示 
三角形第 $j$ 个面片迭代 $t$ 次之后的法向. 上述的网 格上的双边滤波算法是一个局部迭代算法, 它可 以得到较好的去噪效果，但难以解决收玫性问题. 随着迭代次数的增多, 噪声被卷积操作逐步平滑 掉的同时, 不同程度的特征也会被去掉. 滚动指导 法向滤波 ${ }^{[8]}$ 表示为

$$
\boldsymbol{n}_{i}^{t+1}=\frac{\sum_{j \in \boldsymbol{P}_{i}} \boldsymbol{W}_{\mathrm{c}}\left(\left\|\boldsymbol{c}_{i}-\boldsymbol{c}_{j}\right\|\right) \boldsymbol{W}_{\mathrm{s}}\left(\left\|\boldsymbol{n}_{i}^{t}-\boldsymbol{n}_{j}^{t}\right\|\right) \varepsilon_{i j} \boldsymbol{n}_{j}}{\sum_{j \in \boldsymbol{P}_{i}} \boldsymbol{W}_{\mathrm{c}}\left(\left\|\boldsymbol{c}_{i}-\boldsymbol{c}_{j}\right\|\right) \boldsymbol{W}_{\mathrm{s}}\left(\left\|\boldsymbol{n}_{i}^{t}-\boldsymbol{n}_{j}^{t}\right\|\right) \varepsilon_{i j}}
$$

从式(3)可以看出, 滚动指导滤波与双边滤波在权 重设计方面是相似的，主要区别在于卷积作用的 对象由上一步所得面法向变成噪声网格的法向. 滚动滤波算法在每一步迭代中加人原始噪声法向 的目的就是要将原始包含特征信息的法向重新加 人高斯卷积, 以免像双边滤波那样随着迭代进行, 只有上一步的法向信息被保留，而逐步丢失原始 的信息. 滚动滤波在很大程度上可保持模型特征, 但是由于噪声法向的全程参与, 最终结果不可避 免地包含噪声信息. 所以，本文滤波算法希望通过 自适应插值的方式来平衡好噪声网格和磨光网格 在迭代过程中的作用.

同时本文根据双边滤波的高斯卷积过程提出 不同程度特征的概念，高斯卷积过程中取不同的 方差参数 $\sigma_{\mathrm{s}}$, 去掉不同程度的特征信息. 如图 1 所 示, 其中, $a$ 表示原始模型, $b \sim d$ 分别表示高斯函数 中方差参数 $\sigma_{\mathrm{s}}=0.3, \sigma_{\mathrm{s}}=0.5, \sigma_{\mathrm{s}}=1.0$ 的滤波结果. 当 $\sigma_{\mathrm{s}}$ 的值较小时, 双边权重较小, 去掉微弱特征; 反之, 双边权重较大, 去掉大的结构特征. 从图 1 示意图看出，不同的 $\sigma_{\mathrm{s}}$ 取值得到不同程度的特征. 本文的迭代插值算法在迭代插值的过程中与双边 滤波的高斯卷积类似，同样根据高斯卷积通过系 数 $\sigma$ 控制插人不同的特征信息.

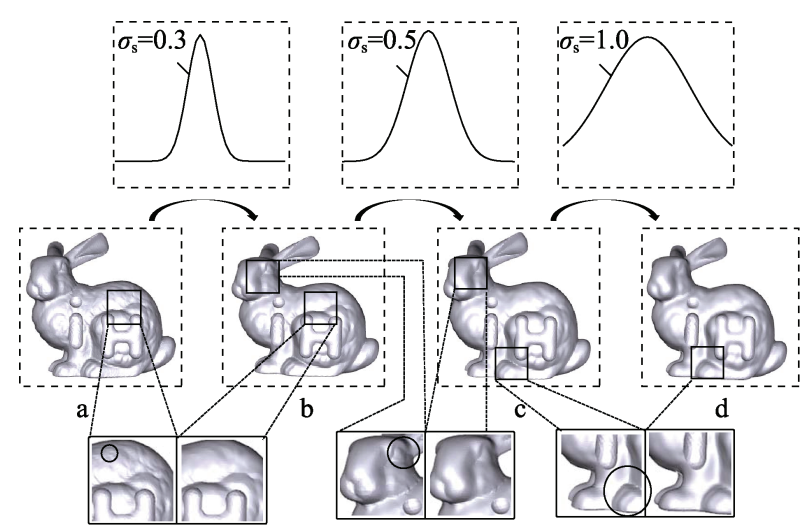

图 1 不同程度特征定义说明图

\section{4 线性插值保特征算法}

为去除噪声, 现有算法往往对模型进行平滑 去噪，而大尺度的平滑往往会去掉模型弱特征，显 然噪声模型虽有噪声, 但其中包含模型的全部特 征与细节信息. 因此, 如何从噪声模型中提取有效 信息是本文算法的核心. 本文的去噪模型应该是 噪声模型和过渡光滑模型两者的中间产物, 因此 提出自适应的插值算法.

\section{1 线性插值过程}

首先, 定义噪声模型为 $\boldsymbol{T}$, 光滑之后的模型为 $\boldsymbol{S}, \boldsymbol{F}$ 表示最终得到的结果模型. 插值的总体的 思想表示为

$$
\boldsymbol{F}=\lambda \boldsymbol{S}+(1-\lambda) \boldsymbol{T}
$$

将上述插值思想转化为以三角面片面法向为载体 的形式表示为

$$
\boldsymbol{N}_{\mathrm{F}}=\lambda \boldsymbol{N}_{\mathrm{S}}+(1-\lambda) \boldsymbol{N}_{\mathrm{T}}
$$

其对每一个三角形面片的法向进行处理. 其中, $N_{\mathrm{T}}$ 表示原始噪声模型的面法向; $\boldsymbol{N}_{\mathrm{S}}$ 表示光滑模 型的面法向； $N_{\mathrm{F}}$ 表示最终模型的面法向； $\lambda$ 是平 衡原始模型和光滑模型的参数. 从式(4)(5)可以看 到插值过程的本质, 就是在原始模型和光滑模型 中通过找到合适的 $\lambda$ 来达到去噪的目的.

\section{2 自适应插值过程}

噪声模型由噪声和原始模型 2 部分组成，虽然 其有一些失真, 但是其中还是保留大量原始模型 的细节. 因此, 本文算法旨在从噪声模型中将原始 模型不同程度的特征逐步地加到充分磨光模型上. 为了在迭代插值过程中设计自适应的插值参数, 将迭代插值过程表示为

$$
\boldsymbol{N}_{\mathrm{F}}^{k+1}=\left(1-\alpha_{k}\right) \boldsymbol{N}_{\mathrm{F}}^{k}+\alpha_{k} \boldsymbol{N}_{\mathrm{T}}
$$

其中, $N_{\mathrm{F}}^{0}$ 的初始值为 $N_{\mathrm{S}} ; \alpha_{k}$ 为自适应参数. 在 设计自适应插值函数时, 在每一步迭代过程中, 主 要考虑如何根据当前步所得面法向与噪声模型面 法向的差异来决定插人噪声网格的特征信息. 为 此，本文引人迭代过程中法向差异

$$
D_{k}=\left\|\boldsymbol{N}_{\mathrm{T}}-\boldsymbol{N}_{\mathrm{F}}^{k}\right\|
$$

根据去噪所需, 本文希望在模型光滑区域 $D_{k}$ 的变 化趋近于零, 此时 $\alpha_{k}$ 也应该接近于 0 , 从而 $\boldsymbol{N}_{\mathrm{F}}^{k} \approx N_{\mathrm{F}}^{k+1}$, 因此只有很少的特征信息需要加人; 而对于特征区域, 本文期望差异 $D_{k}$ 值较大, 稀疏 $\alpha_{k}$ 接近 1 , 即可保持网格的特征. 因为原始法向虽然包 
含真实特征信息，但是也包含噪声. 所以，本文对法 向差异的模增加了做高斯卷积的步骤，从而倾向于 在特征区域插人信息. 随后, 定义自适应插值公式

$$
\alpha_{k}=\varphi\left(D_{k}\right)=1-\mathrm{e}^{-\left|D_{k}\right|^{2} / \sigma^{2}}
$$

将迭代过程中三维数据的法向差异转化为一维数 据的迭代系数，系数 $\sigma$ 控制插人法向的速率. 将 预滤波之后的光滑模型 $N_{\mathrm{S}}$ 作为初始值代人迭代式 (6)中，经过计算和化简可以得到关于光滑模型 $N_{\mathrm{S}}$ 和原始噪声模型 $\boldsymbol{N}_{\mathrm{T}}$ 的式(7), 同时也可以求得系 数 $\lambda_{k}$, 如

$$
\left\{\begin{array}{l}
\boldsymbol{N}_{\mathrm{F}}^{k}=\lambda_{k} \boldsymbol{N}_{\mathrm{S}}+\left(1-\lambda_{k}\right) \boldsymbol{N}_{\mathrm{T}} \\
\lambda_{k}=\prod_{i=1}^{k}\left(1-\varphi\left(\boldsymbol{N}_{\mathrm{T}}^{i}-\boldsymbol{N}_{\mathrm{F}}^{i}\right)\right)=\mathrm{e}^{\left(\frac{-1}{2 \sigma^{2}} \sum_{i=1}^{k} D_{i}^{2}\right)}
\end{array}\right.
$$

式(6)(9)分别称为局部和整体插值公式. 从式(6)可 以看出，在迭代插人过程中迭代系数 $\alpha_{k}$ 也逐渐变 小，但是每一步都在插人特征. 从式(9)来看，随着 迭代过程的进行, 全局插值系数 $\lambda_{k}$ 呈递减趋势, 这说明噪声法向 $N_{\mathrm{T}}$ 占更大的比重来保持特征. 因 此对于迭代过程, 无论从局部来看还是从整体来 看, 模型的特征都在逐渐恢复.

\section{3 插值算法有效性和收敛性证明}

本文算法在光滑模型上逐步将原始模型的特 征插人，最终其会越来越接近原始模型，并且趋于
收玫趋势. 本节将通过理论推导、法向差异曲线以 及视觉差异 3 个方面来证明收玫性的结论. 首先, 可以从式(9)中的 $\lambda_{k}$ 的公式来得到理论上的证明. 因为

$$
\sum_{i=1}^{k} D_{i}^{2} \geqslant \sum_{i=1}^{k-1} D_{i}^{2}
$$

所以当 $N_{\mathrm{F}}^{k+1} \neq N_{\mathrm{T}}$ 时, $\lambda_{k}<\lambda_{k-1}$, 即随着插值次数的 增多，原始法向所占的比重会越来越多，最后直至 趋近于原始法向; 当 $N_{\mathrm{F}}^{k+1}=N_{\mathrm{T}}$ 时, $\lambda_{k}=\lambda_{k-1}$ 迭代 停止. 因此, 迭代算法的输出将会收玫于原始模型 的法向. 图 2 所示为 Bunny 模型迭代插值的视觉效 果变化示意图, 其展示迭代过程中光滑模型逐渐 插人不同的特征后的视觉变化. 可以看出, 插值迭 代的过程中法向差异的模 $\left|D_{k}\right|$ 的值逐渐变小, 而 且在最开始插人模型的结构特征后插人微弱特征, 直至趋于收玫. 由于原始法向受到噪声干扰, $\left|D_{k}\right|$ 也不可避免地受到影响，也就会进一步影响 在光滑模型中所加人的信息. 为此, 本文提出一种 备选方案, 即在迭代过程中可以对 $\left|D_{k}\right|$ 做高斯卷 积, 以保证加人更多的特征信息. 本文在实验过程 中发现，该备选方案可以辅助保持不同程度特征 (可以从视觉效果看出), 但不影响最终去噪结果. 因此可以从视觉效果上得出算法收敛和保持不同 程度特征的结论.

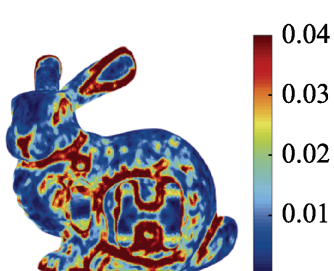

迭代1次

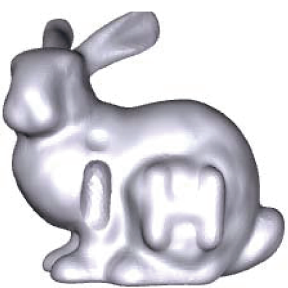

迭代1次

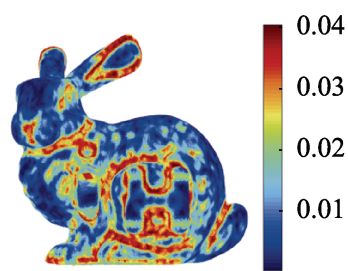

迭代2次 a. 法向差异变化

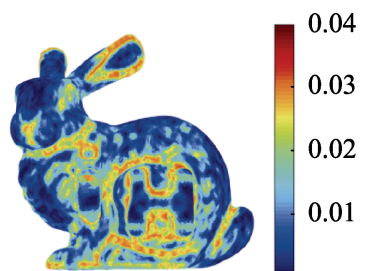

迭代3次

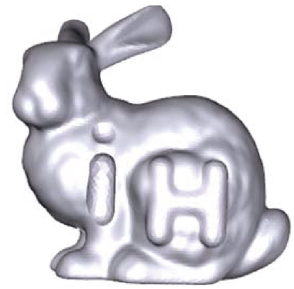

迭代2次

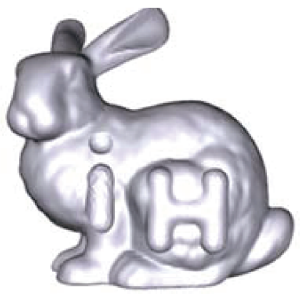

迭代3次

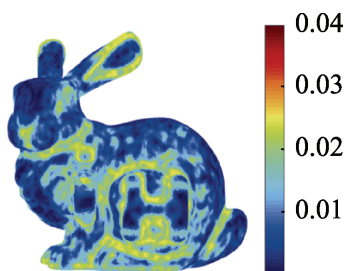

迭代4次

b. 视觉变化

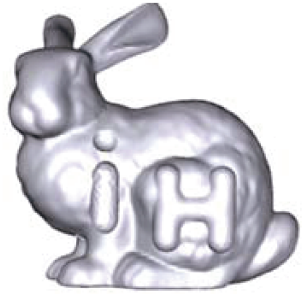

迭代4次

图 2 迭代插值过程变化示意图

同时，本文提出迭代过程中原始法向与迭代 法向的平均差异量

$$
\eta(k)=\frac{1}{n} \sum_{k=1}^{n}\left\|\boldsymbol{N}_{\mathrm{F}}^{k}-\boldsymbol{N}_{\mathrm{T}}\right\|^{2}
$$

图 3 展示了迭代过程中 $\eta(k)$ 的变化趋势, 可 以看出随着迭代数次的增加, 插人特征之后的光 滑模型和噪声模型的差异越来越小, 逐渐趋于收 玫. 差异越小, 迭代过程中插值系数 $\alpha_{k}$ 越小, 插 
人特征的尺度就越小，也就证明本文算法在迭代 过程中是逐渐插人不同程度的特征.

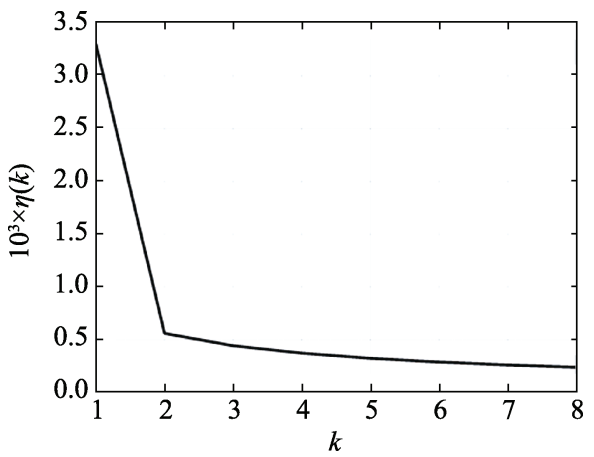

图 $3 \eta(k)$ 迭代变化趋势图

综合本文视觉、迭代过程法向差异变化以及公 式的理论推导可以证明本文算法的收玫性.

\section{4 迭代终止条件}

为了解决三维迭代去噪算法中需人为终止迭 代过程的问题, 更好地从数值角度衡量去噪与保 特征 2 项的关系, 本文提出自动的迭代终止条件, 即目标函数

$$
\begin{aligned}
& \psi(k)=\sum_{k=1}^{n}\left\|D_{k}^{2}(n)\right\|+\beta\left\|\boldsymbol{N}_{\mathrm{F}}^{k}(i)-\boldsymbol{K}_{i} \sum_{j \in N_{i}} \omega_{i j} \boldsymbol{N}_{\mathrm{F}}^{k}(j)\right\|= \\
& \sum_{k=1}^{n} D_{k}^{2}(n)+\beta \sum_{j \in N_{i}}\left\|\boldsymbol{L}\left(\boldsymbol{N}_{\mathrm{F}}^{k}(j)\right)\right\|
\end{aligned}
$$

其分为 2 部分, 第 1 部分为迭代过程中的法向与原 始法向之间的差异项 $D_{k}$, 第 2 部分为基于拉普拉 斯算子 $\boldsymbol{L}$ 的网格平滑性度量. 其中， $\boldsymbol{N}_{\mathrm{F}}^{k}$ 表示上一 步通过迭代插值 $k$ 步后得到整体模型的法向， $\boldsymbol{N}_{\mathrm{F}}^{k}(j)$ 表示第 $j$ 个三角形面片的面法向; 通过上述 2 项来衡量网格去噪保特征程度. 为了研究迭代终 止次数，通过固定插值参数 $\beta$ 来估计最佳的 $k$ 取 值，使用 4 个模型 Fandisk, Octa-flower, Skull, Bunny 进行测试. 在模型上总体代价函数 $\psi(k)$ 的变化趋 势如图 4 所示. 通过观察发现, 当 $k=4$ 或者 $k=5$ 时, 能够得到较小的代价函数值.

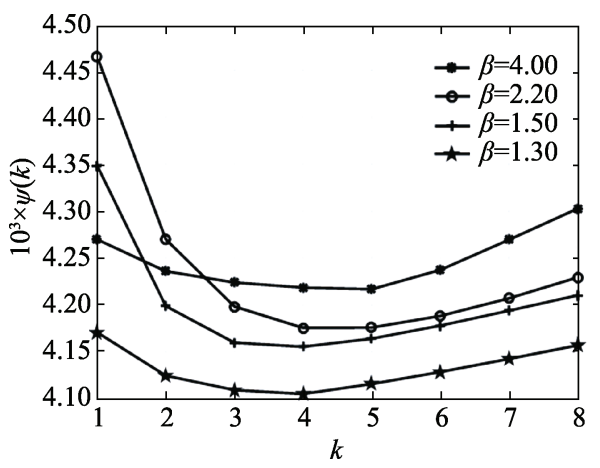

图 4 迭代过程的 $\psi(k)$ 的变化

\section{5 点的位置更新}

在得到去噪的面法向后, 本文采用 Sun 等 ${ }^{[2]}$ 的 算法进行点位置的更新. 根据质心坐标差异和法 向的关系来更新点的位置表示形式如

$$
\boldsymbol{v}_{i}^{\prime}=\boldsymbol{v}_{i}+\frac{1}{\left|\boldsymbol{P}_{\mathrm{v}}(i)\right|} \sum_{j \in \boldsymbol{P}_{\mathrm{v}}(i)} \boldsymbol{N}_{\mathrm{F}}^{k}(i) \cdot\left(\boldsymbol{N}_{\mathrm{F}}^{k}(j) \cdot\left(\boldsymbol{c}_{j}-\boldsymbol{v}_{i}\right)\right)
$$

其中, $v_{i}$ 和 $v_{i}^{\prime}$ 分别为原始点和其更新后的坐标; $\boldsymbol{P}_{\mathrm{v}}(i)$ 和 $\left|\boldsymbol{P}_{\mathrm{v}}(i)\right|$ 分别表示顶点环邻域和其数量; $N_{\mathrm{F}}^{k}$ 表示上一步通过插值更新得到的模型法向; $N_{\mathrm{F}}^{k}(i)$ 表示三角形网格第 $i$ 个网格的法向.

\section{6 本文插值算法}

输人. 噪声模型 $\boldsymbol{T}$, 光滑模型 $\boldsymbol{S}$.

输出. 去噪模型.

Step1. 读人原始模型和光滑模型, 分别得到原始 噪声法向 $\boldsymbol{N}_{\mathrm{T}}$ 和光滑法向 $\boldsymbol{N}_{\mathrm{S}}$

Step2. 将原始法向逐渐插人到光滑法向中去(根据 自动终止条件终止):

Step2.1. 通过式(7)根据在 $\boldsymbol{N}_{\mathrm{T}}$ 与 $\boldsymbol{N}_{\mathrm{F}}^{k}$ 的法向差异 求得 $D_{k}$;

Step2.2. 将法向差异的模做高斯卷积;

Step2.3. 将高斯卷积之后的 $D_{k}$ 值代人到式(8)中 求得 $\alpha_{k}$;

Step2.4. 通过式(6)进行迭代插值.

Step3. 通过式(13)更新点的位置.

\section{5 实 验}

\section{1 实验环境}

本文算法在 $3.70 \mathrm{GHz}$ Intel ${ }^{\circledR}$ Core(TM) i7-8700K CPU 上进行实验, 代码通过 MATLAB 实现. 在不 同的网格模型上使用本文算法，包括噪声程度不 同的合成模型和自然模型, 其中一些甚至是人为 地重新取样来模拟不规则表面取样. 这些模型是 为了验证本文算法对不同程度噪声模型以及不规 则采样的去噪效果. 在算法的时间复杂度方面, 局 部迭代算法相比于其他类型算法时间复杂度较低. 本文插值算法比局部算法更加高效，因为本文算 法在计算插值参数之后，主要进行法向之间的加 法操作，节省大量的时间. 本文算法主要有模板获 取和迭代去噪 2 个耗时环节. 以 Fandisk, Max, Bunny 为例, 本文算法的时间统计如表 1 所示. 其 中, $V$ 表示网格模型顶点个数, $F$ 表示网格模型中三 角形面片的个数. 
表 1 不同算法在各模型上时间对比

\begin{tabular}{lcccccc}
\hline 实验 & \multirow{2}{*}{$\begin{array}{c}* \\
\text { 模型 }\end{array}$} & $(V, F)$ & 局部算 & \multicolumn{3}{c}{ 本文算法时间 $/ \mathrm{s}$} \\
\cline { 5 - 7 } & & 法 $^{[3]} / \mathrm{s}$ & 模板获取 & 迭代插值 & 总时间 \\
\hline Fandisk & $(6475,12946)$ & 0.5784 & 0.2313 & 0.0845 & 0.3158 \\
Max & $(30942,61880)$ & 1.5992 & 0.5297 & 0.5314 & 1.0611 \\
Bunny & $(34834,69451)$ & 2.9048 & 0.7262 & 0.7432 & 1.4694 \\
\hline
\end{tabular}

\section{2 算法鲁棒性分析}

为了验证算法鲁棒性, 分别从带洞模型、非均 匀采样模型和不同程度的噪声模型 3 个方面进行 验证. 如图 5 所示, 本文算法能够有效地去除带洞 模型的噪声, 并且在洞的边缘处保持平滑去噪结 果，保持洞的原始形状而没有发生形变. 对于非均 匀采样模型和不同噪声程度模型, 图 6 中的 Nicolo 模型为左右采样率不同的模型, 分别加上 $0.1,0.2$ 和 0.5 平均长度的高斯噪声, 从实验结果可以看出, 本文算法能够保证模型左右两侧在采样率不同情 况下取得相同的去噪结果. 当然这样的结果跟充 分光滑模型的获取方式有关, 只要充分光滑模型 能处理好不同采样率的网格模型，本文算法亦能 保持其鲁棒性. 同时,可以看到对于不同噪声的模 型，本文算法均能够取得保持不同程度特征的去 噪效果.

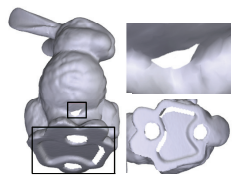

a. 原始模型

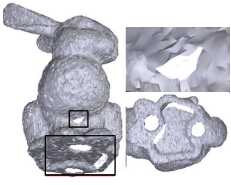

b. 噪声模型

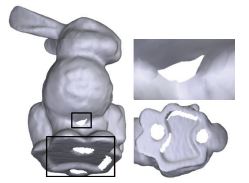

c. 去噪模型
图 5 本文算法带洞模型去噪结果
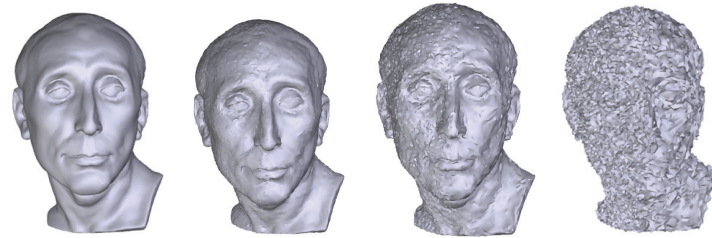

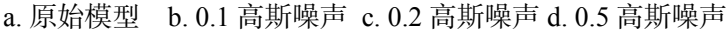
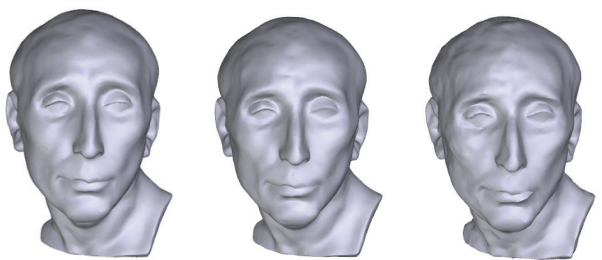

e. b 的去噪结果 f. c 的去噪结果 g. d 的去噪结果

图 6 本文算法同一模型不同采样率去噪结果

\section{3 各种算法对比}

将本文算法与多种经典及流行算法进行比较,
包括双边网格滤波 ${ }^{[1]}$ 、基于鲁棒统计的滤波算法 ${ }^{[24]}$ 、 单边法向滤波 ${ }^{[2]}$ 、指导法向滤波 ${ }^{[4]} 、 L_{0}$ 稀疏算法 ${ }^{[13]}$ 、 全局滤波 ${ }^{[3]}$ 和局部滤波 ${ }^{[3]}$ 、鲁棒高保真的网格去噪 算法 ${ }^{[12]}$. 实验除了全局的算法 ${ }^{[12-13]}$, 其余都是局部 算法. 本文通过比较不同算法处理模型的视觉效果 和均方角度误差值(mean square angular error, MSAE) 来度量法向差异, 其定义为 $\mathrm{MSAE}=E\left[\angle\left(\boldsymbol{N}, \boldsymbol{N}_{\mathrm{F}}^{k}\right)\right]$, 其中, $N$ 为未加噪的原始面法向; $N_{\mathrm{F}}^{k}$ 为滤波所得 面法向; $E[\cdot]$ 代表求期望.

为了进行公平的比较, 调整每种算法的参数 来达到更好的视觉效果. 对于这些算法的参数集 分别是双边网格滤波(顶点迭代次数 $\left.n_{1}\right)^{[1]}$ 、基于鲁 棒统计的滤波算法(空间宽度 $\sigma_{\mathrm{f}}$, 高斯权重影响 $\left.\sigma_{\mathrm{g}}\right)^{[24]}$ 、单边法向滤波(阈值 $T$, 法向迭代次数 $n_{1}$,

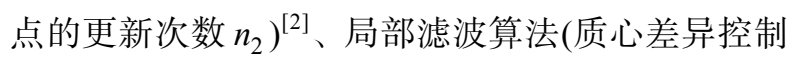
参数 $\sigma_{\mathrm{c}}$, 法向差异控制参数 $\sigma_{\mathrm{s}}$, 法向更新次数 $n_{1}$, 点的更新次数 $n_{2}$ ) $^{[3]}$ 和全局滤波算法 (平衡光滑项 和保真项的系数 $\lambda$, 法向差异控制参数 $\sigma_{\mathrm{s}}$ 点的更 新次数 $\left.n_{2}\right)^{[3],} L_{0}$ 稀疏算法 $\left(L_{0}\right.$ 目标函数的权重 $\lambda$, $\alpha_{0}$ 和 $\beta_{0}$ 表示算法中的初始值, $u_{\alpha}$ 和 $\mu$ 表示 $\alpha$ 和

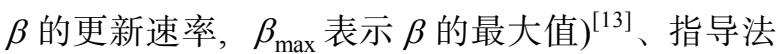
向滤波(几何邻域半径 $r$, 核范围变化 $\sigma_{\mathrm{r}}$, 法向迭 代数量 $k_{\text {iter }}$, 顶点的迭代数量 $\left.v_{\text {iter }}\right)^{[4]}$, 鲁棒高保真 的网格去噪算法(紧密型函数的核宽 $\sigma_{\mathrm{c}}$, 相似性函 数的核宽 $\sigma_{\mathrm{s}}$, 点的更新次数 $\left.v_{\mathrm{iter}}\right)^{[12]}$. 本文算法的 参数主要包含 3 个: 控制插人速率的参数 $\sigma$, 其取 值范围在 $[0.1,3], \sigma$ 越小, 插人的速率就越大; 法 向的迭代次数 $n_{1}$, 根据本文第 4.4 节的结论可以观 察到: 当 $n_{1}=4$ 或 5 时取得最佳效果. 点的更新次数 $n_{2}$ 的取值范围为 $[5,10]$.

\subsection{1 合成模型视觉结果展示}

在定性分析方面，对 5 个去噪常用模型进行去 噪对比. 对 Bunny, Skull, Max 模型加人 0.2 的高斯 噪声, 对 Octa-flower 和 Fandisk 加人 0.1 的高斯噪 声. 对于图 7 图 8 的 2 个合成 Skull 模型和 Bunny 模型，可以看到，Zheng 等 ${ }^{[3]}$ 的局部迭代算法去除 噪声的同时去掉弱特征. 对于微弱细节特征, 如 Skull 模型的牙齿和额头的曲线, 本文算法较其他 算法都将特征保持得更好. 对于 Bunny 模型，可以 看到, 兔子的肌肉和细节在本文算法下得到很好 的保持. 在图 9 的 Max 模型上测试不同采样率下 的去噪情况, 从实验结果可以看到, 在不同的采样 率的模型, 本文算法同样有较好的效果, 如对 Max 
模型的眼部和嘴部细节特征保持得比较细致. 对 于图 10 的 Fandisk 模型, 大多数的算法处理得不 错, $\mathrm{He}$ 等 ${ }^{[13]}$ 算法虽然可以去除噪声, 但是模型边 缘地方会变扁, 本文则在保持细节特征方面有更
多的优势. 对于图 11 的 Octa-flower 模型，与局部 算法 ${ }^{[3]}$ 相比, 本文算法很好地保留模型的边缘特 征; 而文献[12]算法模型恢复得不是很好, 因为其 主要适用于大噪声模型.

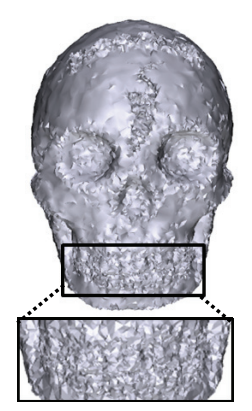

a. 噪声模型

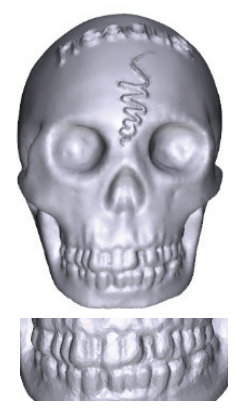

b. 原始模型

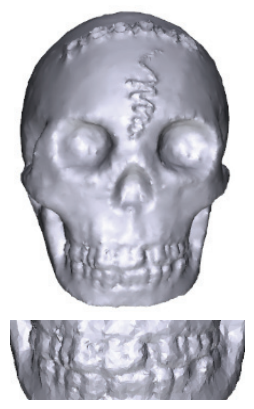

c. 文献[1]

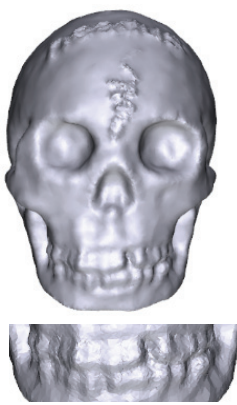

d. 文献[24]

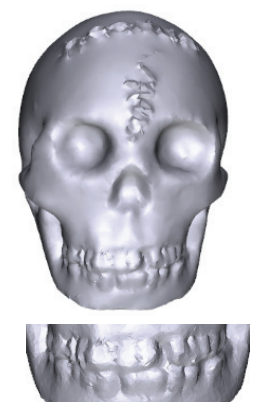

e. 文献[2]

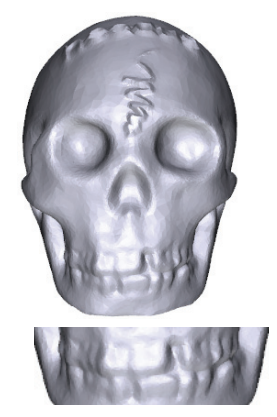

f. 文献[3]局部

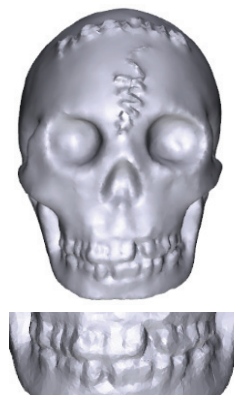

g. 文献[3]全局

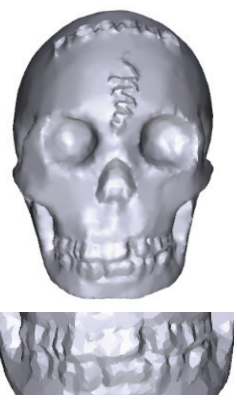

h. 文献 [13]

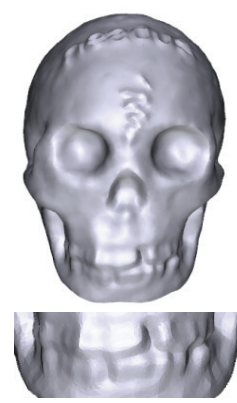

i. 文献[4]

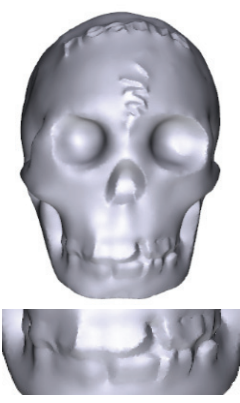

j. 文献[12]

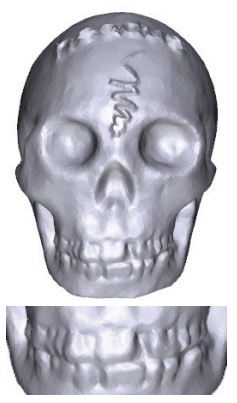

k. 本文

图 7 不同算法对 Skull 模型去噪对比

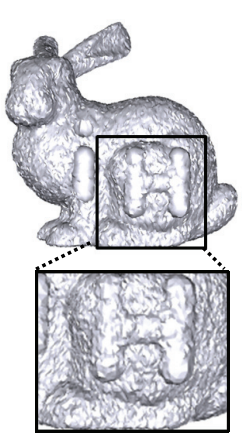

a. 噪声模型

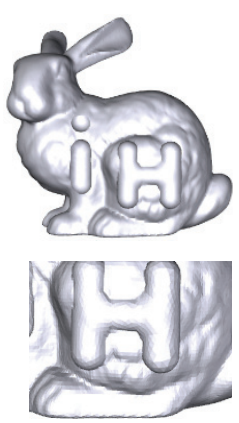

b. 原始模型

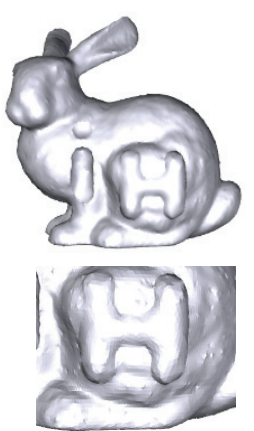

c. 文献[1]

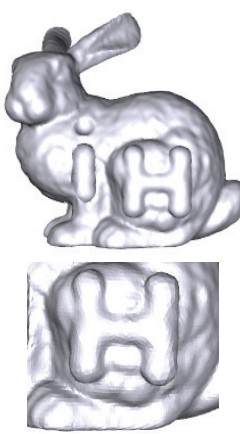

d. 文献[24]

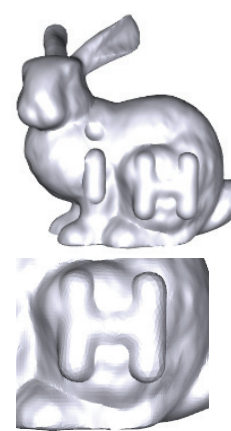

e. 文献[2]

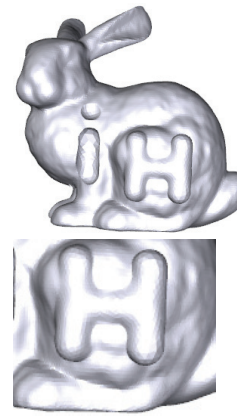

f. 文献[3]局部

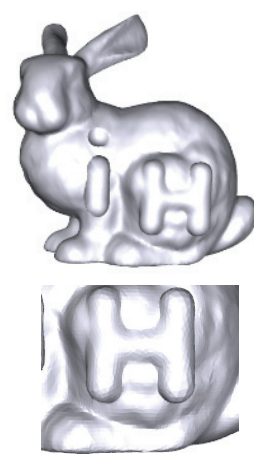

g. 文献[3]全局

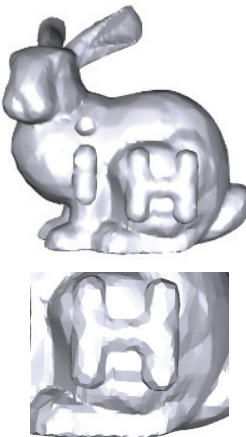

h. 文献[13]

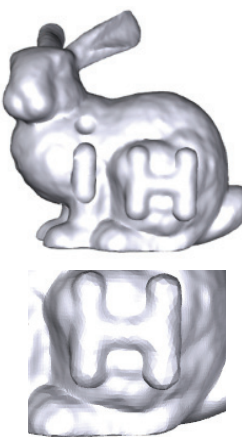

i. 文献[4]

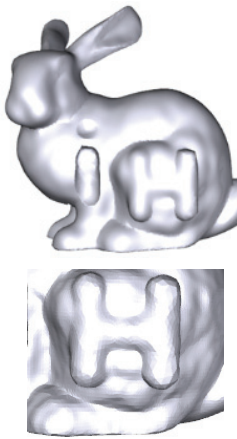

j. 文献[12]

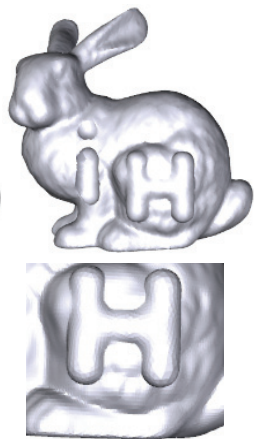

k. 本文

图 8 不同算法对 Bunny 模型去噪对比 


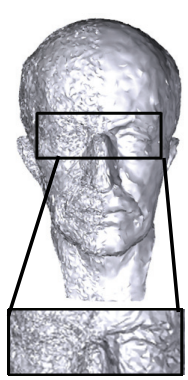

a. 噪声模型

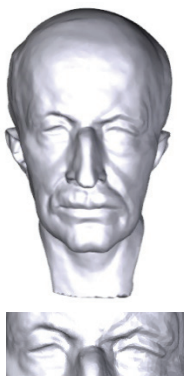

b. 原始模型

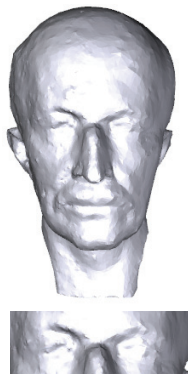

c. 文献[1]

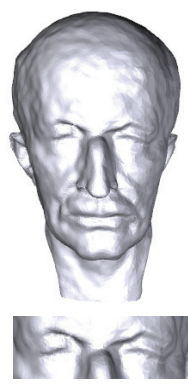

d. 文献[24]
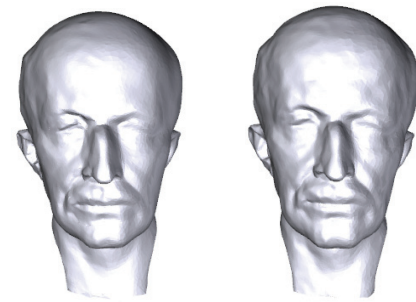

\section{Aird}

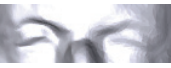

e. 文献[2] f. 文献[3]局部
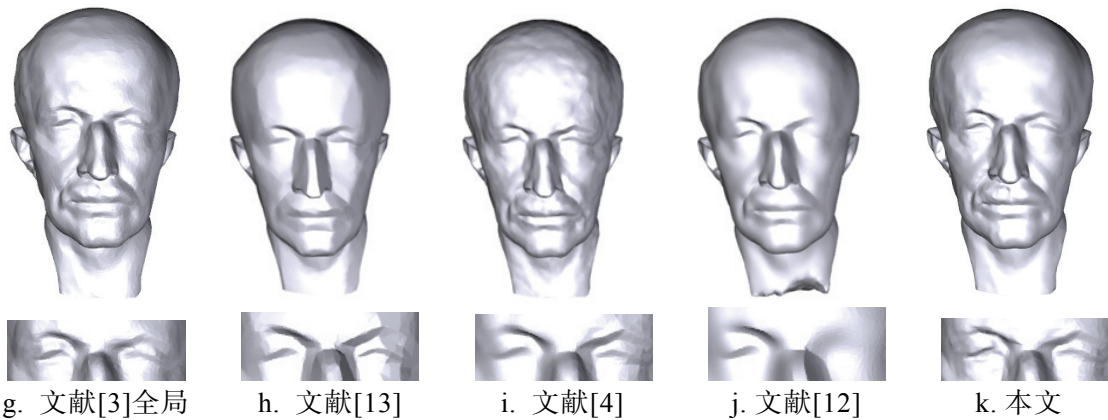

h. 文献[13]

i. 文献[4]

j. 文献[12]

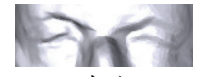

图 9 不同算法对 Max 模型去噪对比

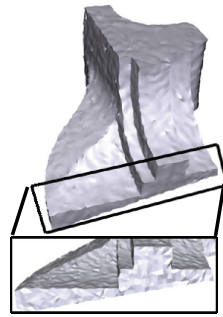

a. 噪声模型

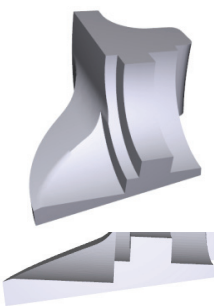

b. 原始模型

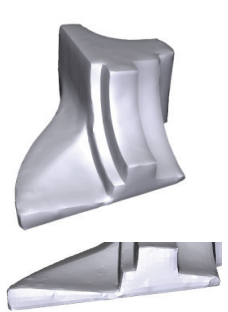

c. 文献[1]

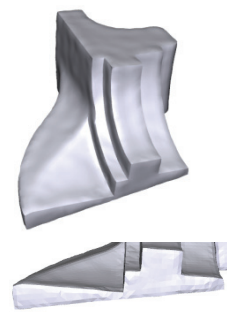

d. 文献[24]

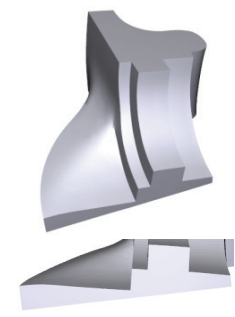

e. 文献[2]

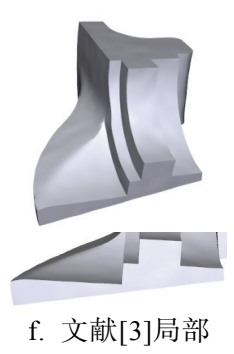

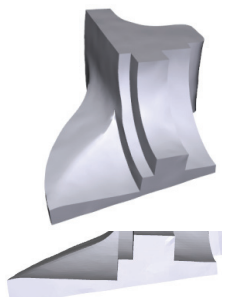

g. 文献[3]全局

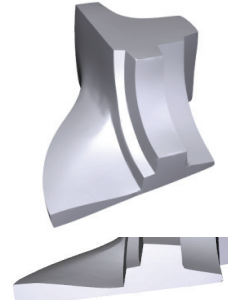

h. 文献[13]

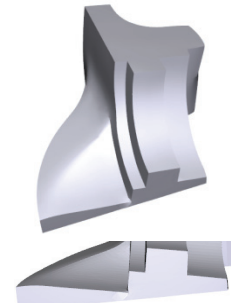

i. 文献 [4]

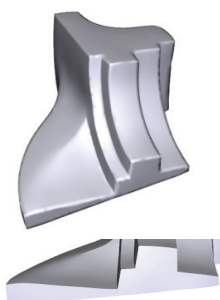

j. 文献[12]

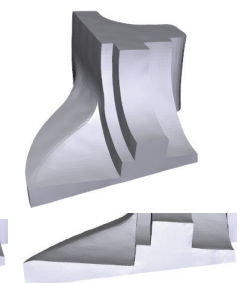

$\mathrm{k}$. 本文

图 10 不同算法对 Fandisk 模型去噪对比

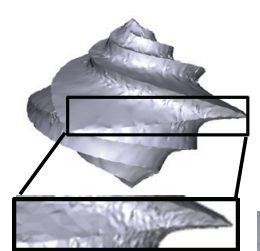

a. 噪声模型

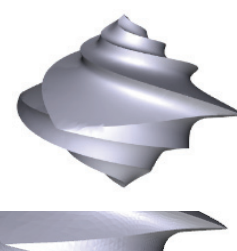

b. 原始模型

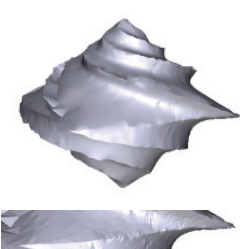

c. 文献[1]

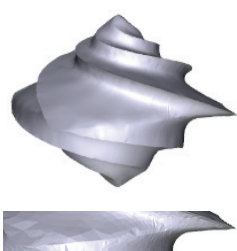

d. 文献[24]

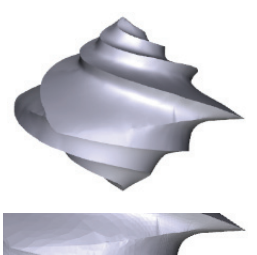

e. 文献[2]

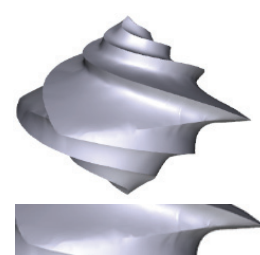

f. 文献[3]局部
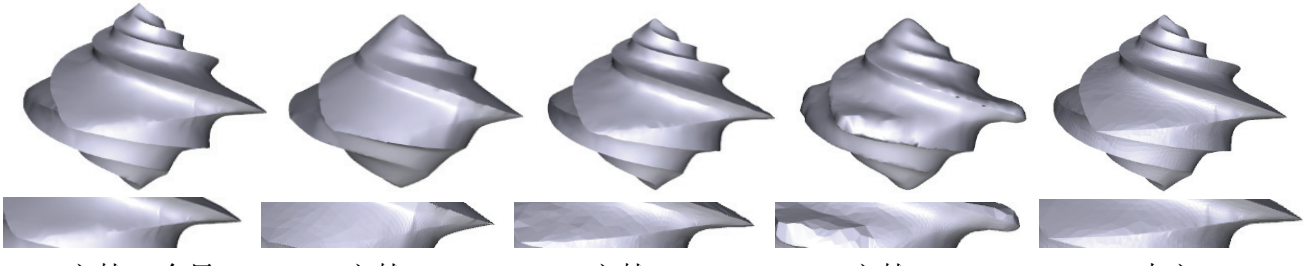

g. 文献[3]全局

i. 文献[4]

j. 文献[12]

k. 本文

图 11 不同算法对 Octa-flower 模型去噪对比 


\subsection{2 真实视觉结果展示}

将本文算法用在真实的扫描模型，如 Angel, Rabbit, Shell 和 Wilhelm. 对于 Angel, 在其原始噪 声的基础上加人 0.2 的高斯噪声, 对于其他模型, 仍然采用其原本的真实数据模型.

通过图 12 图 15 展示的实验结果, 可以看到
本文算法对于真实模型具有较强的鲁棒性. 对于 Angel 模型，本文算法对于细节特征保持得更好， 不仅能够保持 Angel 模型翅膀的主要结构, 还对 Angel 的眼睛的微弱特征保持得比较细致; 同时对 于 Rabbit, Shell 和 Wilhelm 也均有较好的结果. 对 于真实模型, 大多数算法都可以相对较好地去除噪

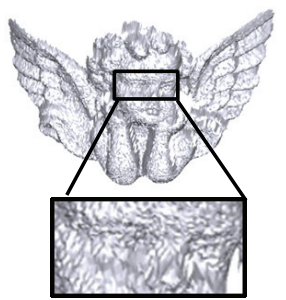

a. 原始模型

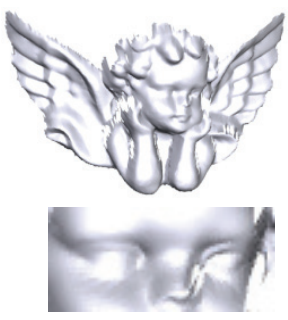

f. 文献[3]全局

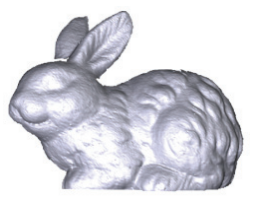

a. 原始模型

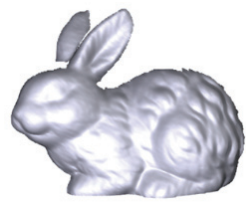

f. 文献[3]全局

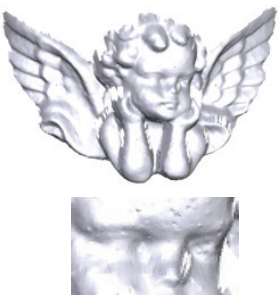

b. 文献[1]

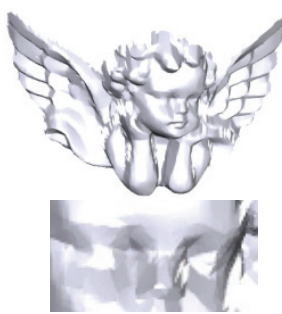

g. 文献[13]

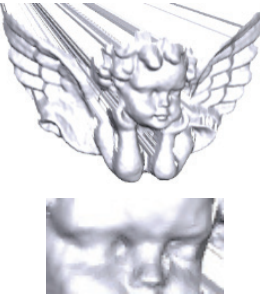

c. 文献[24]

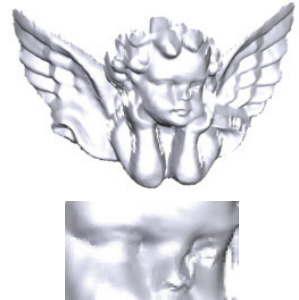

d. 文献[2]

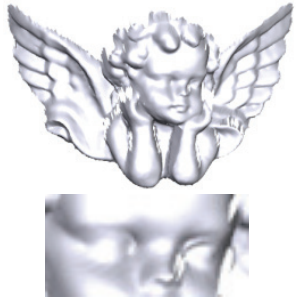

e. 文献[3]局部

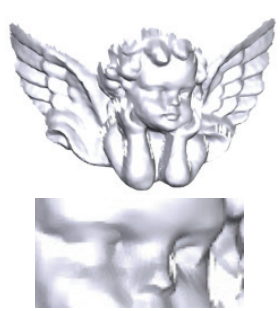

h. 文献[4]

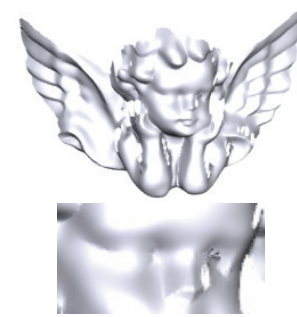

i. 文献[12]

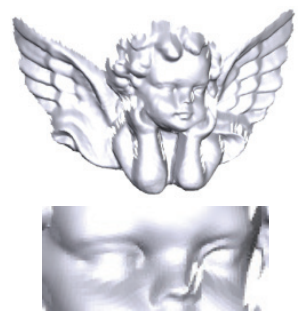

j. 本文

图 12 不同算法对 Angel 模型去噪对比

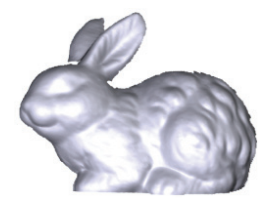

b. 文献[1]

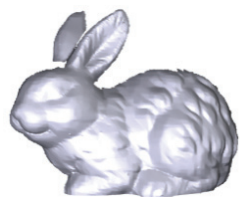

g. 文献[13]

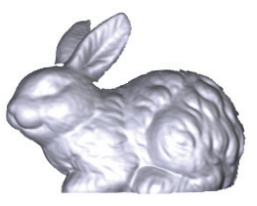

c. 文献[24]

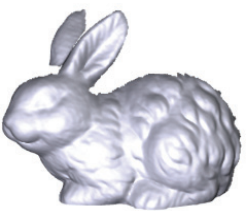

h. 文献[4]

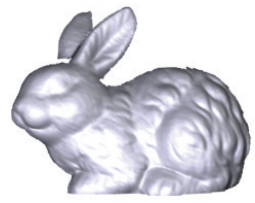

d. 文献[2]

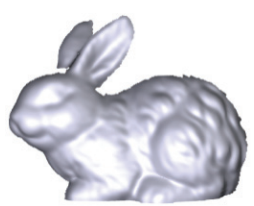

i. 文献[12]

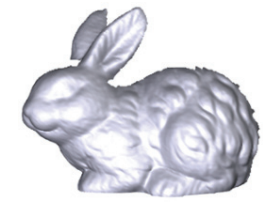

e. 文献[3]局部

图 13 不同算法对 Rabbit 模型去噪对比

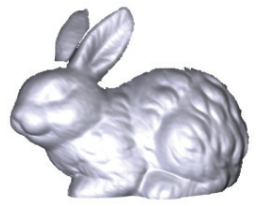

j. 本文

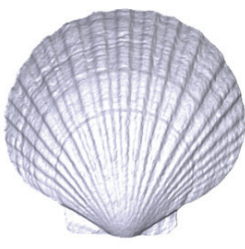

a. 原始模型

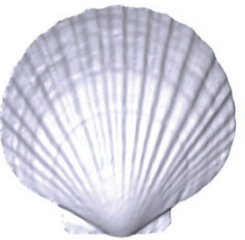

f. 文献[3]全局

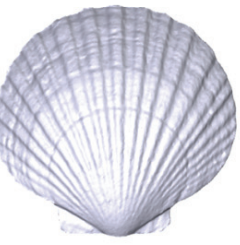

b. 文献[1]

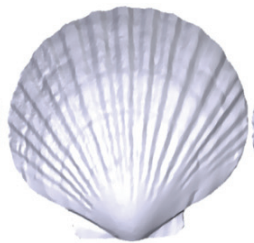

g. 文献[13]

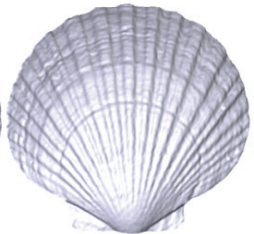

c. 文献[24]

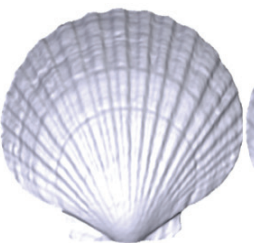

h. 文献[4]

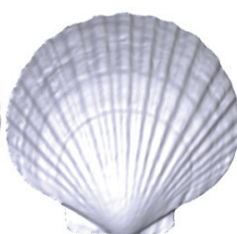

d. 文献[2]

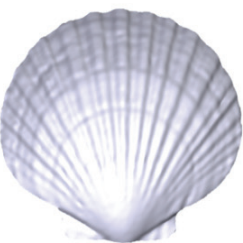

i. 文献[12]

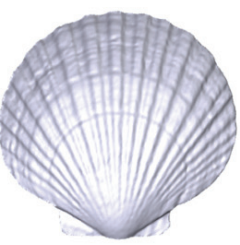

e. 文献[3]局部

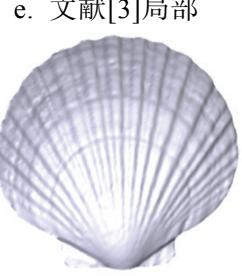

j. 本文

图 14 不同算法对 Shell 模型去噪对比 


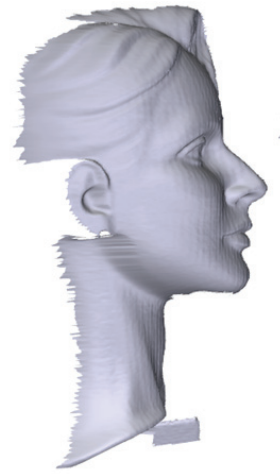

a. 原始模型

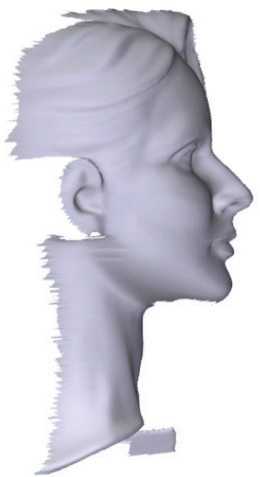

f. 文献[3]全局

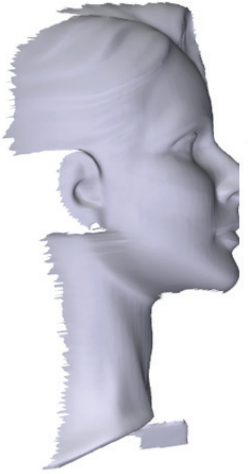

b. 文献[1]

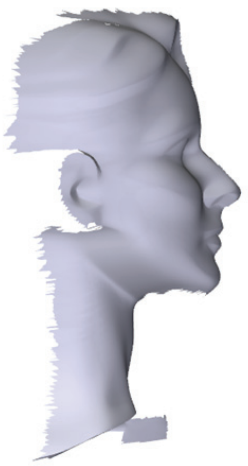

g. 文献[13]

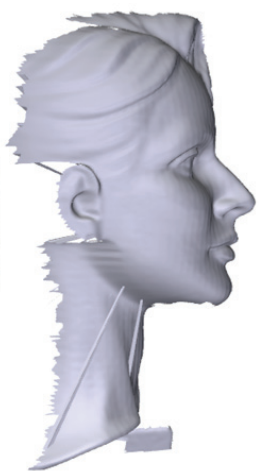

c. 文献[24]

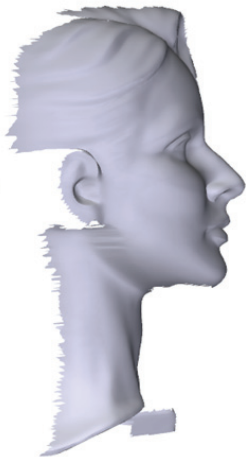

h. 文献 [4]

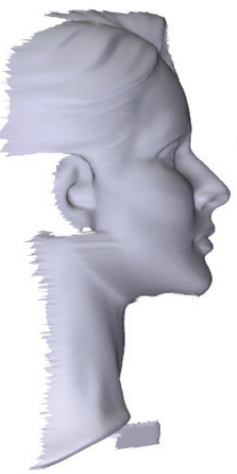

d. 文献 [2]

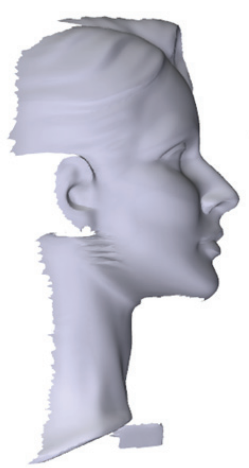

i. 文献[12]

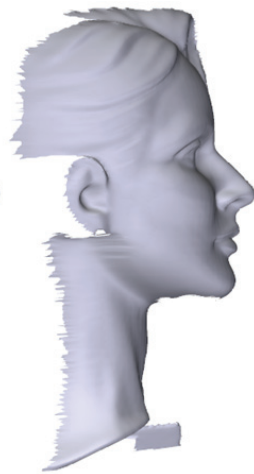

e. 文献[3]局部

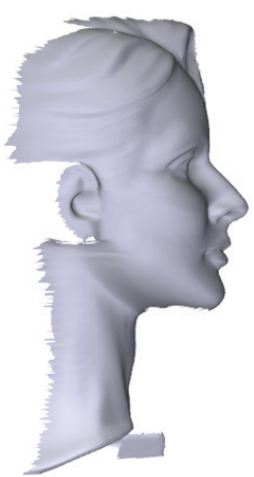

j. 本文

图 15 不同算法对 Wilhelm 模型去噪对比

声，其中文献[24]处理的结果容易有点黏连的情况.

\subsection{3 定量比较与分析}

通过观察表 2 不同去噪算法 MSAE 值可以看
到，本文算法不但在视觉上去噪效果较好，与计算 空间和时间复杂度均较高的算法相比，在定量方 面也可以取得更好或者相当的结果.

表 2 不同去噪算法 MSAE 比较

\begin{tabular}{|c|c|c|c|c|c|c|c|c|c|c|}
\hline \multirow{2}{*}{ 算法 } & \multicolumn{2}{|c|}{ Fandisk $(V=6475)$} & \multicolumn{2}{|c|}{ Octa-flower( $V=7919)$} & \multicolumn{2}{|c|}{$\operatorname{Skull}(V=20002)$} & \multicolumn{2}{|c|}{ iH-bunny $(V=34834)$} & \multicolumn{2}{|c|}{ Max $V=(30$ 942) } \\
\hline & 实验参数 & MSAE & 实验 & ISAE & 实验参数 & MSAE & 实验参数 & MSAE & 实验参数 & MSAE \\
\hline 文献[1] & $(10)$ & 0 & (6) & te & (12) & . & (5) & 0.17071 & $(15)$ & 1555 \\
\hline 文献[24] & $(1.3,1.4)$ & 0.09031 & $(1.8,1.5)$ & 0.11780 & $(1.2,1.6)$ & 10144 & $(1.2,1.5)$ & 0.18260 & $(1.3,1.4)$ & 0.11042 \\
\hline 文献[2] & $(0.55,20,50)$ & 0.03884 & $(0.5,6,20)$ & 0.06790 & $(0.5,6,20)$ & 10279 & $(0.5,5,20)$ & 0.17818 & $(0.35,5,20)$ & 0.09106 \\
\hline 文献[3]局部 & $(0.3,5$, & 0.02478 & $(0.25,6$ & 11 & $(0.5,6,10)$ & 2 & $(0.0, T, 2 v)$ & 0.09209 & $(0.4,4,20)$ & 0.08373 \\
\hline 文献[3]全局 & $\begin{array}{l}(0.3,0.01 \\
10)\end{array}$ & 0.02589 & $(0.1,0.3$ & 05275 & $(0.5,0$. & 91 & 20) & 9723 & $(0.01,0.5,20)$ & 0.08816 \\
\hline 文献[13] & $\begin{array}{l}(0.01,0.003, \\
0.01,0.5, \\
1.414,1000)\end{array}$ & 0.09405 & $\begin{array}{l}(0.01,0.00149 \\
0.001,0.5,1.414 \\
1000)\end{array}$ & 0.31112 & $\begin{array}{l}(0.000004,1, \\
0.001,0.9, \\
1.414,1000)\end{array}$ & 0.12591 & $\begin{array}{l}(0.000004,1, \\
0.001,0.5, \\
1.414,1000)\end{array}$ & 0.18032 & $\begin{array}{l}(0.005,0.002 \\
0.001,0.5 \\
1.414,1000)\end{array}$ & 0.11360 \\
\hline 文献[4] & $\begin{array}{l}(2,0.25,25, \\
20)\end{array}$ & 0.02801 & $(0.35,6,20)$ & 0.07312 & $(2,0.5,6,15)$ & 0.09384 & $\begin{array}{l}(2.7,0.55,4 \\
15)\end{array}$ & 0.19706 & $(2,0.4,4,20)$ & 0.09243 \\
\hline 文献[12] & $\begin{array}{l}(0.5,0.2 \\
100)\end{array}$ & 0.05154 & $(0.55,0.2,100)$ & 0.23132 & $(0.35,0.2,100)$ & 0.12467 & $(0.55,0.2)$ & 0.20332 & $\begin{array}{l}(0.35,0.2 \\
100)\end{array}$ & 0.10876 \\
\hline 本文 & $(0.5,5,10)$ & 0.03789 & $(3,5,20)$ & 0.08341 & $(1,4,10)$ & 12329 & $(1.5,6,6)$ & 0.12599 & $(2,6,8)$ & 0.06787 \\
\hline
\end{tabular}

\section{6 结 论}

本文提出一种简单、高效的自适应插值滤波框 架性算法，其对于细节结构比较复杂的噪声模型处 理效果更佳. 本文算法的本质是在充分光滑模型和 真实噪声模型之间找到合适的插值节点，通过设计
自适应的插值参数和迭代终止条件，很好地对线性 插值过程进行控制. 通过大量的定性和定量的分 析，可以验证本文算法不仅能够移除不同复杂程 度的噪声, 而且能够保留不同程度的特征. 与目前 流行的一系列算法相比，本文算法的计算效率更 高，同时被验证具有快速收敛、鲁棒等优越性质. 


\section{参考文献(References):}

[1] Fleishman S, Drori I, Cohen-Or D. Bilateral mesh denoising[J]. ACM Transactions on Graphics, 2003, 22(3): 950-953

[2] Sun X F, Rosin P L, Martin R, et al. Fast and effective feature-preserving mesh denoising[J]. IEEE Transactions on Visualization and Computer Graphics, 2007, 13(5): 925-938

[3] Zheng Y Y, Fu H B, Au O K C, et al. Bilateral normal filtering for mesh denoising[J]. IEEE Transactions on Visualization and Computer Graphics, 2011, 17(10): 1521-1530

[4] Zhang W Y, Deng B L, Zhang J Y, et al. Guided mesh normal filtering[J]. Computer Graphics Forum, 2015, 34(7): 23-34

[5] Wang P S, Fu X M, Liu Y, et al. Rolling guidance normal filter for geometric processing[J]. ACM Transactions on Graphics, 2015, 34(6): Article No.173

[6] Zheng Y L, Li G Q, Xu X M, et al. Rolling normal filtering for point clouds[J]. Computer Aided Geometric Design, 2018, 62: 16-28ssw

[7] Liu S H, Rho S M, Wang R J, et al. Feature-preserving mesh denoising based on guided normal filtering[J]. Multimedia Tools and Applications, 2018, 77(17): 23009-23021

[8] Li T, Wang J, Liu H, et al. Efficient mesh denoising via robust normal filtering and alternate vertex updating $[\mathrm{J}]$. Frontiers of Information Technology \& Electronic Engineering, 2017, 18(11): 1828-1842

[9] Hurtado J, Gattass M, Raposo A, et al. Adaptive patches for mesh denoising $[\mathrm{C}] / /$ Proceedings of the 31st SIBGRAPI Conference on Graphics, Patterns and Images. Heidelberg: Springer, 2018: 1-8

[10] Centin M, Signoroni A. Mesh denoising with (geo)metric fidelity[J]. IEEE Transactions on Visualization and Computer Graphics, 2018, 24(8): 2380-2396

[11] Liu B, Cao J J, Wang W M, et al. Propagated mesh normal filtering[J]. Computers \& Graphics, 2018, 74: 119-125

[12] Yadav S K, Reitebuch U, Polthier K. Robust and high fidelity mesh denoising[J]. IEEE Transactions on Visualization and
Computer Graphics, 2019, 25(6): 2304-2310

[13] He L, Schaefer S. Mesh denoising via $L_{0}$ minimization[J]. ACM Transactions on Graphics, 2013, 32(4): Article No.64

[14] Zhao Y, Qin H, Zeng X Y, et al. Robust and effective mesh denoising using $L_{0}$ sparse regularization[J]. Computer-Aided Design, 2018, 101: 82-97

[15] Wang R M, Yang Z W, Liu L G, et al. Decoupling noise and features via weighted $\ell_{1}$-analysis compressed sensing[J]. ACM Transactions on Graphics, 2014, 33(2): Article No.18

[16] Zhang H Y, Wu C L, Zhang J Y, et al. Variational mesh denoising using total variation and piecewise constant function space[J]. IEEE Transactions on Visualization and Computer Graphics, 2015, 21(7): 873-886

[17] Liu Z, Zhong S S, Xie Z, et al. A novel anisotropic second order regularization for mesh denoising[J]. Computer Aided Geometric Design, 2019, 71: 190-201

[18] Zhong S S, Xie Z, Liu J Q, et al. Robust mesh denoising via triple sparsity[J]. Sensors, 2019,19(5): 1001

[19] Wang J, Zhang X, Yu Z Y. A cascaded approach for feature-preserving surface mesh denoising $[\mathrm{J}]$. Computer-Aided Design, 2012, 44(7): 597-610

[20] Lu X Q, Deng Z G, Chen W Z. A robust scheme for feature-preserving mesh denoising[J]. IEEE Transactions on Visualization and Computer Graphics, 2016, 22(3): 1181-1194

[21] Xing Y, Bai L, Tan J Q, et al. Robust mesh denoising based on collaborative filters[J]. Journal of Advanced Mechanical Design, Systems, and Manufacturing, 2018, 12(4): JAMDSM0084

[22] Wang P S, Liu Y, Tong X. Mesh denoising via cascaded normal regression[J]. ACM Transactions on Graphics, 2016, 35(6): Article No.12

[23] Wang J, Huang J, Wang F L, et al. Data-driven Geometry-recovering mesh denoising $[\mathrm{J}]$. Computer-Aided Design, 2019, 114: 133-142

[24] Jones T R, Durand F, Desbrun M. Non-iterative, feature-preserving mesh smoothing[J]. ACM Transactions on Graphics, 2003, 22(3): 943-949 